\title{
Formas de participación en línea en estudiantes de la Facultad de Administración de la Universidad Veracruzana en México
}

Forms of Online Participation in Students of the Faculty of Administration of the Veracruzana University in Mexico

\section{Volumen 18, Número 2}

Mayo-Agosto

pp. 1-28

Carlos Arturo Torres Gastelú

Revista indizada en REDALYC, $\underline{\text { SCIELO }}$

Revista distribuida en las bases de datos:

LATINDEX, DOAJ, REDIB, IRESIE, CLASE, DIALNET, SHERPA/ROMEO, QUALIS-CAPES, MIAR

Revista registrada en los directorios:

ULRICH'S, REDIE, RINACE, OEI, MAESTROTECA, PREAL, $\underline{\text { CLACSO }}$ 


\title{
Formas de participación en línea en estudiantes de la Facultad de Administración de la Universidad Veracruzana en México \\ Forms of Online Participation in Students of the Faculty of Administration of the Veracruzana University in Mexico
}

\section{Carlos Arturo Torres Gastelú1}

\begin{abstract}
Resumen: La ciudadanía digital está relacionada con la habilidad de los individuos para participar en la sociedad en línea de manera ética y responsable. Para los fines de este estudio, se parte de la premisa de que las formas de participación en línea constituyen unas de las dimensiones del constructo de Ciudadanía Digital. De ahí que el objetivo de este artículo sea mostrar las formas de participación en línea que realizan los estudiantes de la Facultad de Administración de la Universidad Veracruzana en México. Se trata de presentar los hallazgos de una primera aproximación cualitativa con estudiantes de tres programas educativos: Administración, Administración Turística y Sistemas Computacionales Administrativos, inscritos en la entidad académica seleccionada. Para ello, se desarrolló una guía de entrevista con el fin de identificar las formas de participación en línea que realizan los estudiantes relacionados con su papel como ciudadanos digitales. La técnica de recolección empleada fue la de grupos de discusión, se grabaron las entrevistas, se hicieron las transcripciones y se procedió a realizar la codificación e interpretación de los datos. Los resultados se organizaron en tres dimensiones: Prácticas que realizan como ciudadanos digitales, participación en línea como ciudadano y participación en asuntos públicos a través de Internet. Las evidencias indican debilidades detectadas, que se enmarcan en las escasas acciones que realizan los estudiantes universitarios en su rol como ciudadanos digitales. Como consecuencia, se vis/umbra una ardua tarea pendiente, en el desarrollo de una estrategia educativa, que permita fortalecer la función que debe desempeñar la universidad en el proceso de formación de las personas estudiantes como ciudadanos digitales.
\end{abstract}

Palabras clave: ciudadanía, estudiantes universitarios, participación juvenil, tecnología de la información.

\begin{abstract}
Digital citizenship is related to the ability of individuals to participate in online society ethically and responsibly. For the purposes of this study it is based on the premise that forms of online participation constitute one of the dimensions of the construct of Digital Citizenship. Hence, the objective of this article is to show the forms of online participation of the students of the Faculty of Administration of the Veracruzana University in Mexico. The aim is to present the findings of a first qualitative approach to students of three educational programs: Administration, Tourism Administration and Administrative Computer Systems enrolled in the selected academic entity. To this end, an interview guide was developed to identify the forms of online participation carried out by students related to their role as digital citizens. The collection technique used was the discussion groups, the interviews were recorded, the transcriptions were made and the data was codified and interpreted. The results are addressed organized in three dimensions: Practices carried out as digital citizens, Participation online as a citizen and Participation in public affairs through the Internet. The evidences indicate detected weaknesses that are framed in the scarce actions carried out by university students in their role as digital citizens, so we can see an arduous task pending in the development of an educational strategy that allows to strengthen the role that the university should play in the process of training students as digital citizens.
\end{abstract}

Palabras clave: citizenship, university students, youth participation, information technology.

\footnotetext{
${ }^{1}$ Académico en la Facultad de Administración de la Universidad Veracruzana, México. Doctor en Ciencias de la Administración (UNAM), con Maestría en Ciencias de la Computación, y Licenciatura en Informática.
}

Dirección electrónica: ctorres@uv.mx

Artículo recibido: 19 de noviembre, 2017

Enviado a corrección: 19 de febrero, 2018

Aprobado: 9 de abril, 2018 


\section{Introducción}

La incorporación de las Tecnologías de la Información y Comunicación (TIC) en la vida cotidiana en nuestra sociedad ha propiciado la aparición y uso de herramientas que permiten a los ciudadanos comunicarse y organizarse unos con otros, además de interactuar con el gobierno y las instituciones. En este sentido, las diversas formas de participación en línea de los ciudadanos se convierten en un mecanismo para analizar la incidencia y frecuencia en los asuntos públicos y la implicación social en los procesos de toma de decisiones.

Por su parte, Gómez (2013) señala que estas herramientas tecnológicas favorecen a que los individuos se puedan reunir en comunidades virtuales para compartir información y hacer escuchar sus demandas y necesidades, convirtiéndose en un vehículo de empoderamiento para buscar y exigir respuestas a sus demandas. En primera instancia, sin distinción de clases sociales o políticas, pero si enmarcada por una desarrollada competencia ciudadana digital caracterizada por un mayor compromiso social y la participación cívica.

He aquí donde las instituciones educativas en todos los niveles juegan un rol destacado en el proceso formativo integral del estudiante que dista solo del carácter académico tradicional para inmiscuir ahora el perfil digital del futuro ciudadano. Paulatinamente diversas universidades alrededor del mundo aceptan el hecho de que se requiere un cambio en este momento. Este cambio está relacionado con la función relevante que debe hacer la universidad para contrarrestar la brecha digital que impera en la sociedad a través de su responsabilidad para contribuir en el desarrollo de actitudes, habilidades y conocimientos en los estudiantes. Una de estas competencias claves necesarias a ser desarrolladas es la Ciudadanía Digital.

Para los fines de este estudio se parte de la premisa propuesta por Mossberger, Tolbert y McNeal (2007) los cuales indican que la ciudadanía digital está relacionada con la habilidad para participar en la sociedad en línea y requiere un acceso regular y efectivo a Internet, así como las habilidades para usar esta tecnología. Estos autores dicen que la ciudadanía digital puede ser medida por las actividades políticas y económicas que los individuos realizan en línea. También se considera la postura de Ribble, Bailey y Ross (2004) cuando enfatizan que la ciudadanía digital puede ser definida como el conjunto de normas de comportamiento referente al uso de la tecnología. 
En base a lo anterior, se propone una manera de valorar a la ciudadanía digital basada en la participación en línea que realizan los individuos al estar relacionada con la habilidad que poseen para participar como individuos, así como a las diversas formas de comportamiento que realizan cuando interactúan en línea. Estos aspectos de manera conjunta constituyen una dimensión de estudio de la ciudadanía digital. Cabe señalar que solo se pretende abordar esta dimensión en este documento a pesar de que el constructo de ciudadanía digital es mucho más amplio.

Ahora bien, la aparición de Internet favoreció la conformación de nuevas formas de participación ciudadana a través de las herramientas tecnológicas que habilitan la creación de espacios virtuales que permiten en un primer nivel la socialización entre pares, en un segundo nivel la difusión o réplica de la información, y en un tercer deseable nivel la construcción de comunidades virtuales de tópicos especializados que favorecen la discusión y revisión crítica de una temática.

Este proceso de conformación de la participación en línea ciudadana puede ser abordado desde la perspectiva de la democracia digital. En este sentido, Campos y Silván (2012) plantean dos concepciones fundamentales de democracia: sustantiva y procedimental. La democracia sustantiva se basa en las políticas necesarias para garantizar las libertades a los ciudadanos, de modo que estos puedan ejercer sus derechos. En tanto, la democracia procedimental se centra en la participación activa de los ciudadanos en los asuntos públicos. En este estudio, se aborda esta última perspectiva en términos de identificar la percepción sobre su capacidad de los ciudadanos digitales en formación estudiantes universitarios -, partiendo de las interacciones virtuales que realizan con sus pares, con la sociedad y con las autoridades.

Los esquemas de participación en línea han propiciado el surgimiento de nuevas formas de comunicación digital que contribuyen a democratizar el proceso de comunicación. Uno de los ejemplos más representativos queda manifiesto a través del uso de las redes sociales ya sea por permitir el acceso a los ciudadanos a una gran cantidad de información, o bien como un vehículo promotor de la participación favoreciendo la organización de masas, colaborando a la formación de la opinión pública, o simplemente a través del intercambio de información y opiniones (Saorín y Gómez-Hernández, 2014; Viché, 2015).

En cualquiera de los casos, el Internet y sus diversas aplicaciones se han convertido en el mecanismo masificador de gran envergadura al favorecer que los individuos puedan informarse unos a otros a gran escala (Islas y Arribas, 2010), sin importar el rol que decidan 
desempeñar en la red ya sea como consumidores de información o bien como productores de contenidos.

De acuerdo con Bringué y Sádaba (2011), el uso más habitual que los jóvenes dan a las redes es el contacto y la creación de relaciones, el entretenimiento y el conocimiento de vidas ajenas. De la misma manera, autores como Torres-Gastelú y Moreno Coatzozón (2013) y López (2016) destacan que el uso del Internet por parte de los jóvenes universitarios se orienta más hacia el ocio y el entretenimiento y menos hacia la adecuada explotación de sus potencialidades como un elemento favorecedor en los diversos escenarios de aprendizaje. Aunque esta situación no sólo prevalece a nivel universitario, sino que se presenta desde el nivel básico de educación (Torres-Gastelú y Valencia-Avilés, 2013).

De ahí la relevancia de realizar estudios que ahonden sobre la comprensión de las formas de comunicación mediadas por tecnología, en aras de valorar en qué medida contribuyen al empoderamiento de los jóvenes para el uso completo y extenso de sus potencialidades, o si por el contrario permanecen como un punto pendiente. Autores como Díez-Gutiérrez, Fernández-Rodríguez y Anguita-Martínez (2011) apuestan por que este tipo de tecnologías debieran de fomentar el debate y el ejercicio de una ciudadanía joven activa como un mecanismo cotidiano.

En otro estudio publicado por la Fundación Telefónica citado por Gavilán, MartínezNavarro y Fernández-Lores (2017, p. 62) revela que "existe una amplia disparidad en la participación de los ciudadanos en las redes sociales entre quienes se limitan a indicar que una noticia les gusta, los que las reenvían, los que comentan, hasta los que se implican aportando nuevos materiales". Es evidente la necesidad de desarrollar una cultura digital en los individuos que permita orientar el uso de estas herramientas tecnológicas para empoderar el rol que como ciudadanos pueden ejercer de manera rutinaria. Esta responsabilidad de formación cívica y ética para desenvolverse en la virtualidad vista desde el ámbito educativo debiera iniciar desde temprana edad y finalizar en el nivel universitario.

En este mismo sentido Yuste (2015, p. 183), hace mención sobre la aparente desigualdad en el uso que le están dando los jóvenes universitarios a las redes sociales, dado que se enfocan más en verlas como un vehículo que les permite "expresarse, compartir, informarse, debatir y organizarse en red", y no tanto como una gran oportunidad para "favorecer su formación en el uso de estas redes, sus competencias y el desarrollo de pensamiento crítico". De ahí que valga la pena valorar una posible brecha entre los usos 
cotidianos de las redes sociales con la potencialidad que ofrecen las diversas herramientas tecnológicas en las formas de participación en línea que realizan de manera cotidiana los jóvenes.

El crecimiento exponencial que ha tenido Internet y sus diversas formas de aplicación ha sobrepasado la capacidad de las instituciones educativas para enfrentarse a un cambio que demanda la formación integral ya no de ciudadanos tradicionales, sino de ciudadanos digitales que sean capaces de manifestar sus opiniones en línea en los diversos foros. De ahí la relevancia en realizar estudios que valoren el rol que ha desempeñado la universidad en este proceso formativo, específicamente en relación al nivel de madurez que han logrado desarrollar los estudiantes universitarios en cuanto a las diversas formas de participación en línea.

En este sentido, surge una pregunta que guía el presente documento: ¿Cuáles son las formas de participación en línea que realizan los estudiantes universitarios en su rol como ciudadanos digitales? Para comprender las acciones que realizan los jóvenes en cuanto a las diversas formas de participación en línea se abordan tres preguntas detonantes que les fueron planteadas a los participantes: ¿Qué acciones realiza un ciudadano digital?, ¿Cómo manifiestas tu ciudadanía digital en Internet?, y ¿De qué manera haces valer tu rol como ciudadano digital ante las autoridades?

Esta postura analítica se orienta a valorar el nivel de incorporación de las tecnologías de información en la vida cotidiana de los estudiantes universitarios en relación al rol que desempeñan cuando interactúan en las redes sociales. En este sentido, estudios realizados por Livingstone, Haddon y Görzig (2012), destacan una tendencia en los jóvenes adultos que se ve reflejada en determinadas carencias en el uso de estas tecnologías expresado en la poca actividad que realizan en redes sociales aprovechando la totalidad de sus potencialidades. Estos autores señalan que solo un reducido grupo identifica y aprovecha sus posibilidades, mientras que la mayoría contempla la actualidad informativa como mero observador pasivo.

Una expresión de las potencialidades de participación en línea que pueden atender los jóvenes universitarios, se manifiesta por su capacidad de influencia en las redes sociales. Sin embargo, en el estudio realizado por Gavilan, Martínez-Navarro, y Fernández-Lores (2017, p. 68), encontraron "una reducida capacidad para influir en el entorno, crear agenda y movilizar a la sociedad". Estos autores proponen una tipología de jóvenes representada por 
pro-digitales, moderadores duales y escépticos totales, siendo los primeros los que reflejaron mayor actividad aunque fueron la minoría.

Además consideran estos autores que aquellos estudiantes universitarios que sí logran aprovechar las redes sociales de una mejor manera, no consideran que la universidad haya desempeñado un papel clave en el desarrollo de competencias para explotar estas redes, o bien haya mejorado sus habilidades cognitivas. De hecho, buena parte de los jóvenes universitarios no perciben que el rol de la universidad esté asociado a convertirlos en diestros ciudadanos digitales, a su vez reconocen que "dirigen su presencia en la red principalmente a la socialización más que a la participación en la actualidad" (Gavilan, Martínez-Navarro, Fernández-Lores, 2017, p. 68).

Por otra parte, en un estudio realizado por Cano-Correa, Quiroz-Velasco y NájarOrtega (2017, p. 78) sobre el análisis de la percepción que tienen los jóvenes universitarios limeños sobre la política, medios y participación, encontraron que la mayoría de los jóvenes universitarios mantienen una misma actitud tanto hacia los medios digitales como a los tradicionales, es decir: "que se informan pero no interactúan, y se limitan a seguir, comentar o intercambiar información con sus pares inmediatos, pese a que reconocen estos medios por su potencial interactivo".

Este estudio concluye con la necesidad de que "la Universidad, atendiendo a las críticas de los jóvenes respecto de la política, se constituya en espacio de deliberación y acción pública, como parte de la formación integral de los jóvenes" (Cano-Correa, QuirozVelasco y Nájar-Ortega (2017, p. 78), lo cual es coincidente con la aportación del presente documento en términos de la creciente necesidad de incorporar el desarrollo de la competencia de ciudadanía digital como parte de la agenda en las universidades.

Este aspecto es particularmente relevante si se toma en cuenta de que a pesar de que la información de las TIC ha transformado la vida de las personas, existe un rezago sobre sus potencialidades tanto en el impacto que ha tenido en la educación como en el posible empoderamiento que podría ocasionar a los universitarios (Granados-Romero et al., 2014).

Finalmente se destaca que la literatura revisada se enfoca en señalar la acuciante necesidad de formar a los estudiantes universitarios no solo en el plano profesional sino también en el desarrollo de competencias para desempeñarse como ciudadanos digitales (Ballesta y Guardiola, 2001; Valerio-Ureña y Valenzuela-González, 2011). Por lo tanto, se coincide con diversos autores (Ferrés, Aguaded, y García-Matilla, 2012, citado en Gavilan, Martínez-Navarro y Fernández-Lores, 2017, p. 63) en relación con que se requiere hace un 
ejercicio de reflexión y adecuación en el diseño de los "programas de estudio para que incorporen transversalmente asignaturas vinculadas a la alfabetización mediática que potencien las competencias de la ciudadanía".

\section{Uso de las TIC en estudiantes de la Universidad Veracruzana}

La Universidad Veracruzana inició sus actividades en 1944, está localizada en el Sureste de México con presencia en 26 municipios organizada en cinco campus a lo largo del estado de Veracruz (Xalapa, Veracruz-Boca del Río, Orizaba-Córdoba, Poza RicaTuxpan y Coatzacoalcos-Minatitlán). Cuenta con una matrícula total de 78641 alumnos en 314 programas educativos (8 TSU: Técnico Superior Universitario; 174 Licenciaturas; 132 Posgrado). En tanto el número de profesores de tiempo completo es de 2179 (Universidad Veracruzana, 2016).

Con miras a realizar una contextualización local se considera pertinente hacer una breve revisión sobre los estudios que se han abordado sobre el uso de las Tecnologías de la Información y Comunicación en esta universidad. En los últimos años diversos investigadores (Arras, Torres y García-Valcárcel, 2011; Arras, Torres y Fierro, 2012; Borromeo y Ramírez, 2016; Crovi, 2017; Domínguez, 2016; Dorantes, 2016; GarcíaValcárcel y Arras, 2011; López y Alarcón, 2016; Marini, Jácome y López, 2016; Martínez, 2016; Martínez y Ramírez, 2016; Morales, Olguín y Ramírez, 2016; Torres-Gastelú y ArrasVota, 2011) se han dedicado a dilucidar el rol de las Tecnologías de la Información y Comunicación en el ámbito universitario.

En términos generales el interés de algunos investigadores sobre esta temática emana a raíz de que en 1999 surge el Modelo de Educación Integral Flexible (MEIF) que busca propiciar en los estudiantes de las diversas carreras una formación integral y armónica: intelectual, humana, social y profesional (Arredondo, 1999). El modelo pretendía un cambio radical en la concepción de las tareas docentes haciendo énfasis en el uso de las TIC en el salón de clases y en el tránsito hacia otros ámbitos de enseñanza, mediante la diversificación de las tareas a través del uso de las TIC y el sistema multimodal (Universidad Veracruzana, 2009).

En esta universidad se promueve el desarrollo de competencias dentro de las que se da énfasis a las habilidades tecnológicas de los estudiantes universitarios (Casillas y Ramírez-Martinell, 2015) a través del 100\% de los programas educativos incorporados al MEIF que ofrece la Universidad Veracruzana. De ahí la relevancia en valorar y atender no 
solo el desarrollo de habilidades tecnológicas sino en encaminarse a formar individuos que ejerzan de manera ética y responsable su ciudadanía digital. Aspecto que aún no está contemplado de manera oficial pero que amerita ser considerado a integrar.

El abordaje de los estudios realizados en la Universidad Veracruzana ha sido diverso. En primer término, se encuentran investigaciones que abordaban el análisis de la situación en la que se encontraba el desarrollo de las competencias en TIC tanto en estudiantes como en profesores. Acorde a la acuciante necesidad de fortalecer estas competencias en la comunidad universitaria, estos estudios identifican los niveles de competencia de las TIC desde la percepción de estos actores (Arras, Torres y Fierro, 2012; Arras, Torres y GarcíaValcárcel, 2011; García-Valcárcel y Arras, 2011; Torres-Gastelú y Arras-Vota, 2011).

En segundo término, se encuentran investigaciones que abordan el papel que juegan las TIC en el contexto universitario desde diversas perspectivas entre las que se encuentran la brecha digital, el grado de apropiación tecnológica, capital cultural, trayectorias escolares y desempeño académico de los actores universitarios así como el uso e integración curricular de las TIC en entidades en específico (Alanis, López y Olmedo, 2008; Borromeo y Ramírez, 2016, Casillas y Ramírez-Martinell, 2015; Domínguez, 2016; Dorantes, 2016; López y Alarcón, 2016; Marini, Jácome y López, 2016; Martínez, 2016; Martínez y Ramírez, 2016; Morales, Olguín y Ramírez, 2016; Ramírez-Martinell y Casillas, 2015).

En tercer término, se encuentran estudios realizados por investigadores externos en contextos interdisciplinarios que analizaron y explicaron la importancia que los jóvenes atribuyen al uso de las tecnologías digitales en los procesos de expresión, interacción y participación social, con el fin de identificar y sistematizar las características de tales procesos, así como las condiciones de su emergencia. Las cuatro categorías centrales analizadas en estos estudios fueron: juventudes, interacción, cultura digital y redes sociales digitales (Crovi, 2017; Domínguez y López, 2015; López, 2016; López y Anaya, 2016).

Ahora bien, en el contexto de la Universidad Veracruzana, López (2016) señala que en la práctica cotidiana escolar es cada vez más recurrente el uso de las tecnologías, como las redes sociales digitales, por parte de directivos, académicos y estudiantes para construir espacios de colaboración académica. Sin embargo, aún no se ha documentado acerca del cambio tecnológico en la vida de la comunidad estudiantil. Se sabe que las redes sociales digitales son espacios alternativos de expresión y discusión sobre temas de su interés como pueden ser actividades lúdicas y de entretenimiento, prácticas de socialización, recuperar comunicación con personas del pasado o establecer nuevas relaciones. 
Sin embargo, tal como sostiene López y Anaya (2016, p. 50) "ante un uso tan diverso no hay claridad acerca de los temas que les interesan, cómo es su participación, interacción o expresión, así como el potencial de las redes para el desarrollo o ejercicio de una ciudadanía democrática". Como se aprecia, los intereses de investigación actuales en esta universidad se están alineando para contemplar no sólo que los estudiantes puedan ser hábiles con las herramientas tecnológicas, sino que sean hábiles con las diversas formas de socialización digital permitiendo una activa participación como ciudadanos digitales.

Este nuevo enfoque a la realización de estudios sobre las implicaciones del uso de las TIC en los espacios universitarios se justifica en virtud de los pocos trabajos existentes que abordan la relación que guardan las diversas formas de interacción virtual con los medios tecnológicos que usan los estudiantes universitarios en torno a la socialización dentro de los espacios virtuales para denotar el activismo, la participación política o democrática o simplemente el uso de su voz en la red expresada en opiniones, propuestas, quejas o solicitudes tanto dentro del ámbito universitario como fuera de él.

En este sentido, el estudio realizado por López (2016) a estudiantes de la ciudad de Xalapa de la Universidad Veracruzana refiere un pobre ejercicio de la ciudadanía, ya que en lugar de hacer uso de los recursos expresivos que brindan estos canales digitales se conforman con repetir contenidos que alguien más hace públicos. Los jóvenes también tienen una fuerte sensación de que la participación política ciudadana conlleva responsabilidad e información, algo que tal vez no estén dispuestos a asumir. En el caso específico de esta universidad, los hallazgos reiteran estas tendencias, lo cual resulta preocupante porque parece indicar que la política es sólo asunto de unos pocos, mientras la mayoría esquiva esas responsabilidades de participación (Crovi, 2017).

Los avances preliminares que se presentan en este artículo se enmarcan en los procesos de apropiación que han realizado los estudiantes universitarios a través de las mediaciones tecnológicas que emplean de manera rutinaria, influenciados por un proceso de formación educativa en el que se encuentran inmersos - su formación universitaria - con el propósito de dilucidar los tipos de interacción que realizan en la red en su rol como ciudadanos digitales.

Cabe destacar que en la universidad en que se realizó este estudio no existe un proceso formal de formación como ciudadanos digitales, pero sí un proceso formativo de competencias digitales enfocadas al uso de herramientas tecnológicas tanto dentro del aula como fuera del aula a través de la realización de las tareas y proyectos. De ahí la relevancia 
de este estudio al permitir detectar las formas de participación en líneas existentes con miras a proponer estrategias educativas que permitan fortalecer el comportamiento ético que debe imperar en un ciudadano digital responsable.

\section{Metodología}

Los avances de la investigación reportadas en este artículo se enmarcan en el proyecto de investigación "La ciudadanía digital desde la perspectiva del estudiante universitario" registrado en la Universidad Veracruzana y financiado por el CONACYT (Consejo Nacional de Ciencia y Tecnología) a través de la convocatoria de estancia sabática en su edición 2017.

El objetivo que persigue este proyecto es conocer el nivel de manifestación ciudadana digital que ejercen los estudiantes universitarios. Sin embargo, en este documento se presentan resultados parciales que provienen del primer acercamiento que se tuvo con un grupo de estudiantes universitarios a manera de estudio exploratorio durante la primera fase del proyecto. En este sentido, en este artículo se limita a mostrar las formas de participación en línea que realizan los estudiantes de la Facultad de Administración de la Universidad Veracruzana en México, a manera de avance en el estudio exploratorio quedando para otro momento profundizar sobre los resultados finales del proyecto.

De ahí que se recuperen las evidencias cualitativas obtenidas mediante la aplicación de una guía de entrevistas a estudiantes de la Facultad de Administración en la Universidad Veracruzana localizada en el estado de Veracruz en México. Para ello se seleccionó a los tres programas educativos que tuvieran más de 15 años de egresados: Administración, Administración Turística y Sistemas Computacionales Administrativos.

Cabe señalar que el alcance a mediano plazo del estudio incluye la participación de al menos cuatro facultades de dos regiones geográficas en la Universidad Veracruzana (Veracruz y Xalapa). Así como a estudiantes de otra universidad: Instituto Tecnológico de Sonora (ITSON) lo cual permitirá contrastar las percepciones entre el alumnado del norte y sur de México.

Sin embargo, para los fines de este artículo se optó por seleccionar a la Facultad de Administración ubicada en la Ciudad de Veracruz de la Universidad Veracruzana, en virtud de que el autor inició el trabajo de campo en esta entidad debido a que está adscrito a dicha facultad como académico. 
La estrategia metodológica inicial consistió en la revisión bibliográfica de descriptores clave: ciudadanía digital, jóvenes universitarios, participación en línea y participación digital en distintas bases de datos científicas. Posteriormente, con objeto de acercarnos al objeto de estudio se diseñó una guía de entrevistas para orientar el diseño de instrumentos cuantitativos y orientar el rumbo de la investigación.

Por tanto, se decidió usar la técnica de recolección de datos de los grupos de discusión o grupos focal, debido a que es una técnica cualitativa que recurre a la entrevista realizada a todo un grupo de personas para recopilar información relevante sobre el problema de investigación. Se consideró pertinente para obtener las percepciones sobre el área de interés del estudio de manera colectiva. Con ello se perseguía inducir a la activa participación de los estudiantes seleccionados, minimizando las respuestas cortas puesto que se seleccionaron a estudiantes que eran compañeros, amigos o al menos conocidos por estar estudiando la misma licenciatura y el mismo semestre.

Tal como señala Ibañez (1998, citado en Crovi, 2017, p. 25, quienes

participan en un estudio que se apoya en los grupos de discusión se integra al proceso de investigación como sujeto en proceso, ya que mediante la conversación expresa sus opiniones y puntos de vista. El diálogo reivindica al sujeto y sustituye el esquema clásico pregunta-respuesta, propio de la mirada cuantitativa.

Precisamente, eso se requería para la etapa en la que se encontraba el desarrollo del proyecto, contar con un mecanismo para acercarse a los sujetos de estudio y con ello comprender un poco más el mundo virtual en el que se desenvuelven.

En los grupos de discusión participaron 17 alumnos inscritos en el séptimo semestre durante el periodo Agosto 2016 - Enero 2017. Para ello, se realizaron 3 grupos de discusión. El primero con 7 alumnos de la licenciatura en Administración, el segundo con 5 alumnos de la licenciatura en Administración Turística, y el tercero con 5 alumnos de la licenciatura en Sistemas Computacionales Administrativos. El criterio de selección de los informantes consistió en invitar a participar a 5 estudiantes de cada licenciatura en 3 salones al azar, de ahí que no se consideró al género como factor de estudio, sino al interés por contribuir en el estudio. La edad mínima de los informantes fue de 21 años.

La elección de los informantes que cursaban semestres avanzados (7 de 8 semestres a nivel licenciatura en la mayoría de las universidades públicas de México) obedece al interés por conocer las percepciones de estudiantes próximos a egresar, partiendo de la 
premisa de que ellos posiblemente estarían un poco más concientizados acerca de lo que implica ejercer la ciudadanía digital en la sociedad del conocimiento.

La guía de entrevistas empleada incluye a 3 preguntas detonantes y 14 preguntas específicas que abordan aspectos sobre las acciones que ejecutan los estudiantes como ciudadanos digitales, las formas de manifestaciones de su ciudadanía digital en la Internet, así como las maneras en que hacen válida su ciudadanía con las autoridades.

Para facilitar el análisis de la participación en línea se organizaron tres dimensiones de estudio (Prácticas que realizan como ciudadanos digitales, Participación en línea como ciudadano, y Participación en asuntos públicos a través de internet. Cada una de las dimensiones fue presentada a los participantes de los grupos de discusión mediante una pregunta detonante (¿Qué acciones realiza un ciudadano digital?, ¿Cómo manifiestas tu ciudadanía digital en internet?, y ¿De qué manera haces valer tu rol como ciudadano digital ante las autoridades?).

Previo al proceso de grabación de las entrevistas se les explicó a los alumnos seleccionados el objetivo del trabajo y se les pidió su consentimiento y autorización para hacer uso de sus comentarios vertidos en el proceso de análisis y posterior publicación de los hallazgos. Cabe señalar que todos los participantes aceptaron e incluso permitieron que se mencionara tanto su nombre como la licenciatura que cursaban. De ahí que, los nombres de los informantes, así como las licenciaturas que se enuncian a lo largo del texto en cada una de los testimonios son reales.

El procesamiento de datos incluyó la grabación de las 3 sesiones de los grupos de discusión con el previo consentimiento de los participantes, cada sesión duró aproximadamente una hora. Posteriormente se llevó a cabo la transcripción literal de las grabaciones, se trabajó con las tres dimensiones propuestas organizando la información en categorías, y se generó la interpretación de los hallazgos.

\section{Evidencias cualitativas}

A continuación se presentan los resultados organizados en base a las dimensiones de estudio propuestas para analizar la participación en línea que realizan los estudiantes universitarios. 


\subsection{Prácticas que realizan como ciudadanos digitales}

En relación con la dimensión sobre las prácticas que realizan como ciudadanos digitales se plantearon aspectos sobre el conocimiento y ejercicio de sus derechos y obligaciones como ciudadanos digitales, el rol que ha desempeñado la universidad en su formación como ciudadano digital y su percepción con respecto a la forma como ejerce su ciudadanía digital.

Los estudiantes universitarios identifican su rol como ciudadanos digitales por el simple hecho de estar conectados a Internet como un mecanismo para mantenerse en comunicación y para obtener información: "...la forma como uso Internet más que nada es para informarme acerca de un tema que no sepa, así me informo...y me sivve también para compartir ideas con mis compañeros...". (Joshua- Estudiante de Administración).

La mayoría de los informantes señalan que tienen conocimiento sobre los derechos y obligaciones como ciudadanos digitales. Sin embargo, cuando se les pidió que explicaran sobre ello sus opiniones tomaron diferentes rumbos. Algunos se enfocaban en la protección de datos personales: "...yo creo que en el momento en el que nosotros nos registramos en una página, y damos nuestros datos personales, es decir información personal, pues estamos ejerciendo nuestra ciudadanía porque ya estamos dando nuestras referencias más exactas inclusive como para encontrarnos de una manera más fácil...". (Luis - Estudiante de Administración Turística). Detonando ligereza y escasa preocupación sobre el asunto: "... hasta la fecha no existen reglas que rigen esto y espero que no las exista porque el Internet siempre ha sido libre y espero que lo sea..." (Luis - Estudiante de Administración Turística).

Por otra parte, los estudiantes universitarios consideran que ejercen su ciudadanía digital de manera positiva: "...lo que yo hago es que cuido siempre la integridad, cuido lo que comparto, creo que hay que ser muy cuidadoso... pues quieras o no el internet no es una fuente muy confiable al cien por ciento...". (Monserrat - Estudiante de Administración Turística). Sin embargo, hubo evidencias de que no todos tienen los debidos cuidados: "...pues de forma negativa se podría decir porque estamos dando información pero sabemos que no es cien por ciento segura, pero no estamos haciendo así como que muy cuidadoso con nuestra información...." (Joshua - Estudiante de Administración).

Los alumnos de semestres avanzados claramente identifican que no se deben afectar a terceras personas como una forma de expresión positiva de su ciudadanía digital. Otra manifestación positiva de la ciudadanía tiene que ver con compartir información relevante a 
sus círculos sociales, compañeros y demás. Además, consideran vital el respeto hacia los demás y hacia uno mismo como un elemento clave que permite la convivencia digital sana.

En tanto las formas que identifican como manifestaciones negativas que pueden sufrir como ciudadanos digitales se asocian a los llamados delitos cibernéticos como son el robo de identidad, de datos personales y datos bancarios, secuestros, extorsiones y demás. A pesar de que se identificaron a sí mismos como actores con un comportamiento positivo en lo referente a las formas como manifiestan su ciudadanía digital, es meritorio señalar que las evidencias apuntan a un desconocimiento acerca de los derechos y obligaciones que contraen como ciudadanos digitales (Torres-Gastelú, Angulo-Armenta, Lagunes-Domínguez y Soto-Ortiz, 2017).

Dentro de los aspectos que reconocen ampliamente en su mayoría tiene que ver con el derecho al acceso a la información y a la libre expresión como elementos vitales a ejercerse como ciudadanos digitales. Además, se constata un sentido crítico referente a la forma correcta de desempeñarse en las redes siguiendo reglas de etiqueta, así como el cuidado de su imagen digital a través de medidas de seguridad cuando navegan en Internet.

Los entrevistados señalan que evitan a toda costa las agresiones, los tratos rudos o altisonantes, o bien simplemente molestar a otras personas en Internet. Pareciera haberse desarrollado una conciencia sobre un desarrollo armónico en el mundo virtual que contrasta con el creciente índice de amenaza a la seguridad en el ciudadano común y corriente en la sociedad mexicana.

Otra muestra en la conformación de su madurez en las acciones que realizan los estudiantes cuando participan en línea se asocia con el cuidado de la imagen digital, eventualmente desarrollada poco a poco a medida que pasa el tiempo. Diversas evidencias apuntan que lo aprendieron por ensayo y error hasta que ocurrió un evento que los hizo reflexionar sobre los riesgos y afectaciones que conllevan publicaciones impulsivas.

La gran mayoría de los participantes señaló que fue precisamente a través de la interacción cotidiana en las redes sociales donde se llevó a cabo este aprendizaje. Particularmente, Facebook se convirtió en el mecanismo de identidad y presencia virtual en el cual se dio una acotación en el desenvolvimiento de los estudiantes pasando de una apertura total, pública y poco crítica a otra mesurada, cerrada a sus círculos de amigos y más prudente. También, se manifiesta en la privacidad de sus datos personales, los cuales consideran que es crucial y procuran evitar publicar datos que los identifiquen por temor e inseguridad del posible uso que les daría gente con malas intenciones. 
Están conscientes de que el Internet al ser de carácter público se convierte en un riesgo latente si no se toman las debidas precauciones. A pesar de ello, la mayoría no leen las condiciones y políticas de uso en las aplicaciones y plataformas tecnológicas que utilizan. Algunos de los informantes se decantaron en reconocer que las características de las condiciones legales son extensas, difíciles de leer, aburridas y concuerdan en que solo leen una pequeña parte (Torres-Gastelú, Angulo-Armenta, Lagunes-Domínguez y Soto-Ortiz, 2017). De ahí que existe un bajo interés en conocer a detalle las largas y complejas cláusulas que se solicitan para instalar aplicaciones o ser aceptadas en las redes sociales.

Se evita publicar cosas sin que se haya confirmado con diversas fuentes. Saben que una vez publicado todo el mundo lo verá y por ende adoptan una postura responsable reflejado en la mesura, certeza y reserva de lo que publican. Particularmente estas actitudes están presentes en aquellos que mantienen un mayor grado de participación al manifestar sus opiniones en la red sobre diversos temas. Se procura cuidar lo que publican en aras de evitar controversias o malos entendidos.

Reconocen que el estilo de publicación está regido por la persona a la que le están publicando. No lo hacen igual entre sus amigos cercanos que con aquellos que mantienen contacto pero no tanta confianza. Equiparan el trato virtual con el que hacen de manera física. En este sentido, demandan que el trato que le de otra persona sea igual al trato que ofrecen. Aseguran que se vincula con una forma de comunicación respetuosa.

Respecto al rol que ha desempeñado la universidad en su formación como ciudadano digital, se manifiesta en su carácter formativo que se ha concentrado en incorporar las TIC en el proceso de enseñanza-aprendizaje: "...la universidad me ha estado cambiando es la forma de ver Internet porque cada vez se necesita más el internet, de una u otra manera aunque no lo queramos ver así... de hecho pues antes entrar a la universidad yo casi no subía tareas o cosas así casi todo escrito o en papel impreso y ahora casi todo es mediante el internet, yo envió mis tareas, reviso mis archivos y demás...de hecho ya ni siquiera busco libros o sea ya casi todo lo buscó en internet, busco libros en internet de hecho..." (Joshua Estudiante de Administración Turística).

Sin embargo, el rol de la Universidad en la formación de una ética digital se considera como una tarea cubierta de manera parcial: "...creo que en la universidad, lo único que han hecho es de cierto modo recordarme los maestros lo que ya sé, porque yo siento que la escuela no tiene por qué encargarse de eso, más bien la escuela simplemente... y más a nivel universitario se encarga de tu formación profesional, es más bien de saber que está 
bien y que está mal, ahí es cuestión de ética o sea, si hay algunos maestros que entre las pláticas nos recalcan que es lo que está bien, que es lo que está mal..." (Daniel - Estudiante de Administración).

Algunas de las evidencias de los entrevistados apuntan en el mismo sentido con los resultados obtenidos en el estudio realizado por Gavilan, Martínez-Navarro, Fernández-Lores (2017, p. 69) cuando señalan que no es razonable que el grupo de estudiantes que parecía más diestro "en el manejo de las redes sociales, el más participativo y el que con más probabilidad disfrute del beneficio asociado al empoderamiento, aparentemente considere que la universidad en la que se está formando es ajena a esta capacidad".

Por otra parte, algunos informantes consideran que la Universidad si ha ayudado en el proceso de formarlos como ciudadanos digitales puesto que los han acercado a familiarizarse con una gama de herramientas y aplicaciones tecnológicas que les permite comprender que así deben desempeñarse en el futuro cercano como ciudadanos: “...aquí en la universidad nos han dado las herramientas para ejercer más nuestra ciudadanía digital, como las aplicaciones que manejamos para hacer trámites, inscribirnos, ver nuestras calificaciones..." (Melissa - Estudiante de Sistemas Computacionales Administrativos).

\subsection{Participación en línea como ciudadano}

La segunda dimensión referente a las formas de participación en línea como ciudadano plantea aspectos sobre los tipos de manifestaciones de las interacciones virtuales que han realizado en redes sociales, consideraciones sobre la prudencia y mesura en lo que publican, nivel de madurez ético en la navegación, así como su rol ante el consumo y producción de contenidos.

Referente a si los estudiantes universitarios han manifestado su opinión en algún medio de comunicación masivo, las evidencias señalan que llegan a publicar sus comentarios principalmente en Facebook, y lo restringen a su círculo de amigos: “...he llegado a publicar en Facebook, respeto a temas políticos, a los problemas que hemos tenido por ejemplo con el nuevo presidente en nuestro país ... hace algún tiempo si publicaba cosas referentes a que no estamos bien en México... referente igual a la homosexualidad porque sí estoy a favor de este movimiento..." (Susana - Estudiante de Administración Turística). 
En este mismo sentido Ohme, Albaek, y de Vreese (2016:143) destacan que a pesar de que los jóvenes reconocen "un uso intensivo de las redes sociales, particularmente de Facebook, no se corresponde necesariamente con una mayor exposición a información política o bien participación política".

El Facebook se constituye como la herramienta tecnológica por excelencia al regular la vida virtual del estudiante, pero que se restringe a un círculo típicamente cerrado de amigos en donde el pre-requisito es conocerlo físicamente. Se mantiene poca apertura a incluir a nuevos miembros en sus redes que no se tenga contacto previo. Por tanto, se tiene una clara conciencia de evitar aceptar en sus redes a personas nuevas que intenten contactarlos.

En relación con la gestión de la privacidad, el mundo de los universitarios gira entorno a las redes sociales como Facebook. Sostienen que les preocupa mucho mantener un ámbito privado con respecto a lo que publican, circunscrito a solo sus amigos en el Facebook. Sin embargo, algunos manifestaron ser más relajados en su seguridad en otras redes como: Instagram o Twitter (Torres-Gastelú, Angulo-Armenta, Lagunes-Domínguez y Soto-Ortiz, 2017).

En términos generales, los entrevistados se sienten confiados en el medio virtual en el que se desarrollan, en parte porque involucran a sus redes a personas que conocen físicamente y eso les da un cierto grado de confianza y seguridad. De ahí que la participación en línea de los estudiantes universitarios se limita a las redes de amigos y frecuentemente se manifiesta a través de memes o alusiones festivas, chistosas o incluso en tono de burla sobre el suceso ocurrido. A partir de ello, se mantiene una constante vigilancia en torno a los esquemas de seguridad en los sitios virtuales donde se interactúa para tener la certeza de quienes son los que tienen acceso a lo que publican.

De manera paralela, se ha desarrollado una conciencia colectiva en relación con la prudencia y mesura en la publicación de sus comentarios en medios de comunicación masivos, en parte por el temor a las implicaciones posteriores: “...no recuerdo alguna ocasión en la que haya dado mi opinión en algún medio de comunicación masivo... tal vez en alguna noticia. Porque a mí sí me da algo de temor que lleguen a tomar represalias por simplemente dar mi punto de vista, entonces procuro no hacerlo. Aunque, si hay una manera en la que pueda participar, que realmente sé que va a ayudar a alguien, entonces lo hago, pero sino simplemente no doy mi opinión pública..." (Alexa - Estudiante de Administración Turística). 
Aunque cabe destacar que este proceso de madurez en la navegación en las redes sociales se da como un producto de la experiencia a través del paso del tiempo. Algunos estudiantes les toma años comprender lo riesgoso que es estar expuesto en el mundo virtual. En este sentido, parece que aún les queda camino por recorrer en cuanto al nivel de madurez sugerido para desempeñarse como ciudadanos digitales, en virtud de que no les queda claro a todos, el posible daño que les cause lo que publiquen en Internet. $\mathrm{O}$ bien lo intuyen pero les toma tiempo asimilarlo y aún más incorporar buenos hábitos en su quehacer diario.

Las manifestaciones de sus opiniones como ciudadanos digitales son escasas. Vinculan su rol casi exclusivamente en términos de sus redes sociales y en un ambiente relajado, poco crítico y sin afán de hacer alguna contribución a la sociedad. Se visualiza su participación en línea como una extensión de su vida social en sus círculos inmediatos, pero no se alcanza a vislumbrar el potencial de su voz en el ámbito virtual.

En algunos casos, los estudiantes señalaron que más que ser productores de contenido, se consideran como consumidores de contenido y su labor se limita en compartir algo que ya estaba publicado en la red: “... cuando yo veo algunas noticias generalmente trato de no dar mi opinión, pero sí compartirla por cuestiones de seguridad y así evitar represalias, uno nunca sabe que pueda pasar o sea cómo otras personas puedan tomar tu comentario y por algo tan insignificante pues se pueda crear un problema mayor..." (Mayra Estudiante de Administración Turística).

Los estudiantes se convierten en mecanismos socializadores de lo que ocurre en su entorno: “...depende de la relevancia del tema, si es algo que es muy importante y que realmente la sociedad lo necesita saber y estar informada, si debería compartirlo, pero sí es algo trivial no tiene caso..." (Montserrat - Estudiante de Administración Turística). Esta apreciación coincide con la detectada en el estudio de Cano-Correa et al. (2017) cuando sostienen que "los universitarios, en general, aprecian más la oportunidad de acceder a información con prontitud que la posibilidad de emitir información".

El mecanismo de participación imperante en los entrevistados se caracteriza por compartir publicaciones previas a sus contactos, y no tanto por manifestar sus opiniones, por lo que se presentan características de consumidores de información y no tanto de productores de información. De ahí que se pueda deducir que el nivel de participación queda expresado como espectador, ya que les gusta saber qué ocurre en la sociedad, pero mantienen discreción respecto a sus opiniones y los medios que ocupan. Resultados 
similares fueron encontrados por Cano-Correa et al. (2017) dado que las prácticas de los jóvenes universitarios limeños están vinculadas fundamentalmente al consumo y no se constituyen ellos mismos en protagonistas a pesar de que "reconocen de manera unánime que las redes sociales posibilitan la comunicación horizontal y la democratización de la opinión".

Tienen dificultad para visualizar el potencial de su participación como ciudadanos digitales, su actuación se circunscribe al entretenimiento, al ocio y a la socialización (TorresGastelú y Moreno-Coatzozón, 2013; López, 2016). En este sentido, algunos estudiantes manifestaron que este tipo de actuación tiene que ver con un sentido de protección personal de su imagen digital.

A pesar de ello, reconocen que la activa participación en línea es enriquecedora porque les permite construirse un mejor criterio sobre una temática. Se convierte en una oportunidad de reflexionar sobre su forma de pensar y frecuentemente a través de estas interacciones se transforma en nuevas concepciones que conforman una perspectiva diferente generando una visión más completa sobre la temática en cuestión.

\subsection{Participación en asuntos públicos a través de Internet}

La tercera dimensión sobre la participación en asuntos públicos a través de internet plantea aspectos sobre las experiencias previas de los estudiantes universitarios en el apoyo a iniciativas solidarias en línea, su posición frente a demandas o peticiones en línea, así como su interés en participaciones en línea sobre temas políticos.

En relación a la participación en asuntos públicos a través de internet, algunos entrevistados expresaron apoyar iniciativas solidarias mediante la firma de una iniciativa solidaria: “...Sí apoyo ese tipo de iniciativas pero solamente en base a lo que están proponiendo y los posibles beneficios que se obtendrían... no lo haría tanto por si me cae bien el político o cosas así... no! porque pues finalmente yo creo que tienes que tomar los datos necesarios, y no tanto si te cae bien o no... lo relevante es qué es lo que va hacer..." (Liliana - Estudiante de Administración).

En esta categoría el único aspecto en el que los estudiantes manifestaron una actitud positiva se relaciona con el apoyo a iniciativas solidarias mediante su firma digital: "...si he compartido mi opinión por ejemplo cuando se trata de debatir algunas reformas de la reforma energética y demás, incluso firmé ahí algunas iniciativas de change.org, aunque no estoy 
seguro si sirve de algo. Sin embargo, creo que es una forma de expresar mi ciudadanía digital..." (Ali - Estudiante de Administración).

Esta evidencia es acorde con los hallazgos del estudio realizado por López (2016) a estudiantes de la misma Universidad Veracruzana, pero en la región de Xalapa, en términos de la disposición que manifestaron los encuestados para participar activamente tanto en las redes sociales como en sitios o páginas que albergan iniciativas virtuales. Es posible que su disponibilidad está asociada a que dicha participación no requiere necesariamente la presencia física en un determinado momento y que puede concretarse mediante un clic, un comentario o una firma en páginas diseñadas para tal efecto (como change.org), las cuales ofrecen posibilidades de solidarizarse o cooperar con ciertos temas que los convocan.

A pesar de que los estudiantes se manifiestan abiertos a apoyar diferentes tipos de iniciativas, no establecen un seguimiento sobre la causa que apoyaron, ni tampoco se llegan a enterar sobre el resultado de dicha iniciativa: “...Si, si he firmado en varias ocasiones, es muy interesante pero nunca termino sabiendo si hacen lo que se propuso..." (Nahomy Estudiante de Sistemas Computacionales Administrativos). Aunque también se encontraron evidencias de que se favorecen las participaciones altruistas que buscan mejorar deficiencias en el entorno cuando viven las carencias y buscan expresarse o bien participar en movimientos para mejorar la situación local.

Algunos de los estudiantes expresaron su interés por apoyar iniciativas para la preservación del medio ambiente o bien para la conservación de especies animales: "...si estoy interesado cuando se trata de problemas ambientales o bien para salvar a animales, no me interesa cuando se trata de personas..." (Karla - Estudiante de Administración Turística). Esta evidencia coincide con los hallazgos de Delgado-Salazar y Arias-Herrera (2008, p. 293) sobre los problemas que les preocupan a los jóvenes actualmente vislumbrando algo más que sus propios intereses personales, y adoptando otras visiones del mundo que emanan de su amplio acceso a los medios de comunicación.

En este mismo orden de ideas, los hallazgos de López (2016) indican que algunos de los jóvenes que participaron en este estudio hicieron hincapié en que no les gusta abordar temas de índole social en las redes sociales, debido que no les parece el espacio más indicado para hacerlo, y que muchas veces la información que ahí encuentran no es del todo veraz. Sin embargo, dentro de las temáticas que sí les interesa abordar en las redes sociales digitales están los problemas ambientales, violencia infantil, y en general sobre los problemas actuales de la sociedad. 
La actuación de los entrevistados sobre demandas o peticiones adopta posturas de interés personal, y no tanto por atender cuestiones comunitarias, quedando evidente una personalidad individualista en su actuar digital. En relación con la interacción con organizaciones, identifican contactarse a través de las páginas de las empresas para expresar sus quejas y sugerencias. Aunque realmente son pocos los que lo hacen. Posiblemente, esto se deba a una cantidad relativamente limitada de sitios en línea a los que parecen acceder de manera cotidiana los estudiantes de esta universidad (Crovi, 2017).

Por otra parte, la participación en línea en intereses políticos es limitada, en parte para evitar conflictos o discusiones en línea. Se evidencia un alto sentido de discreción en asuntos públicos, en parte propiciado por el miedo a represalias o bien para mantener una situación armoniosa en la interactividad virtual.

\section{Conclusiones}

En términos generales, los estudiantes universitarios mantienen una cierta reserva hacia la participación en línea como ciudadano digital, en parte debido a la incertidumbre e inseguridad que aqueja a la sociedad mexicana. Diversas evidencias se orientan hacia un uso pasivo, discreto, reservado en su rol como ciudadano digital. A pesar de ello, los jóvenes acostumbran emitir comentarios preferentemente en sus círculos sociales cercanos, parecen preocuparse por cuidar quienes son los que pertenecen a su círculo social virtual. En relación con su nivel de involucramiento es aspectos sociales, mostraron interés por los grandes temas y sucesos cotidianos que aquejan al país, pero no participan en sectores abiertos.

Las manifestaciones de las opiniones de los estudiantes como ciudadanos digitales son escasas. Ellos ligan su rol como ciudadanos digitales casi exclusivamente en términos de su participación en sus propias redes sociales en un ambiente relajado, poco crítico y aparentemente sin un deseo de hacer alguna contribución a la sociedad. Su participación en línea es visualizada como una extensión de su vida social limitada a sus círculos inmediatos de conocidos y amigos, quedando restringida vislumbrar el potencial de su voz en el entorno virtual.

El nivel de participación que manifiestan los estudiantes se puede considerar en su mayoría como espectadores. A ellos les gusta conocer que es lo que ocurre en la sociedad, pero mantienen discreción en cuanto a sus opiniones y los medios que ocupan para expresarlas. Las acciones de los entrevistados en relación con las demandas o peticiones en 
línea que realizan adoptan una postura orientada hacia el interés personal, y no tanto en atender a temas comunitarios, siendo evidente una personalidad individualista que se refleja en la forma como actúan digitalmente.

Por otra parte, la participación en línea en cuanto a intereses políticos es limitada, en parte para evitar conflictos o discusiones en línea. Hay evidencia de un alto sentido de discreción en cuanto a temas públicos debido al temor a represalias o bien por el deseo de mantener una situación armoniosa en la interactividad virtual.

Los estudiantes identifican que han tenido una evolución en los últimos 3 o 4 años que les ha permitido ser más maduros en las formas en que interactúan socialmente en la virtualidad. Específicamente acerca de lo que ellos dicen, cómo lo dicen y a quién se lo dicen. En el mismo sentido, parece que han desarrollado un esquema selectivo de depuración referente a cuándo y cómo se manifiestan en Internet.

Finalmente, los estudiantes universitarios enfatizan el rol que ha desempeñado la universidad en su proceso de formación, y reconocen el desarrollo de habilidades y conocimientos adquiridos. Aunque en buena medida se enfocan en la adquisición de competencias tecnológicas relacionadas con un conjunto de herramientas tecnológicas y no tanto a la conformación de un nivel de madurez en su rol como ciudadanos digitales.

De ahí que se coincida con lo señalado por Gisbert y Esteve (2011:54) cuando resaltan el hecho de que "sería deseable que la propia institución universitaria pusiera en valor su contribución a la formación del estudiante desarrollando estrategias adecuadas que permitan asegurar que los estudiantes desarrollan la competencia digital durante su etapa formativa".

Una posible y viable estrategia institucional que ayude a incrementar la influencia que ejerce la universidad en el proceso de formación ético de sus estudiantes con miras a convertirse en ciudadanos digitales puede enfocarse en al menos dos rubros. En primer término, en fortalecer las competencias técnico-pedagógicas en sus profesores a través del Programa de Fortalecimiento Académico (ProFA) orientado en incrementar el uso de las TIC con fines escolares y académicos entre los profesores y los estudiantes a través de las redes sociales al constituirse como uno de los vehículos de amplia difusión entre el estudiantado.

Existen evidencias de un uso discreto de las redes sociales con fines académicos en algunos sectores de la universidad (López, 2017), sin embargo, a pesar de la directriz institucional hacia un amplio uso de las TIC en el aula, persiste un número significativo de profesores que aún no incorporan a las redes sociales como un recurso de comunicación, ni como un recurso pedagógico en sus asignaturas. 
En segundo término, se propone la revisión o creación de programas de estudio específicos que atiendan a la concientización y el desarrollo de actitudes y habilidades en los estudiantes respecto al rol que deben desempeñar como ciudadanos digitales. Respecto a la revisión de los programas de estudio, es sugerente iniciar con la selección de asignaturas transversales en la mayoría de las licenciaturas como puede ser Fundamentos de Derecho o bien alguna de las experiencias de tronco común en todas las licenciaturas del modelo MEIF de la Universidad Veracruzana, para que incluya tópicos relacionados con los buenos hábitos y acciones de un ciudadano digital.

Por otra parte, es viable para el contexto universitario diseñar como formación electiva una asignatura denominada Formación Cívica Digital que se encargue de desarrollar conciencia, actitudes y habilidades sobre el comportamiento ético tecnológico que debe poseer un individuo para desenvolverse como ciudadanos digitales responsables.

Por tanto, permanece como una tarea pendiente el fortalecimiento de un comportamiento ético para con ello lograr que los estudiantes universitarios se conviertan en ciudadanos digitales responsables. De ahí que el rol que ha desempeñado la universidad requiere ser revisado con objeto de tomar medidas sobre el proceso formativo. Es meritorio que este proceso formativo luche contra la inequidad social y las diferencias culturales que prevalecen en la sociedad buscando el desarrollo equilibrado de competencias digitales. De manera particular, se requiere generar estrategias que fortalezcan la alfabetización, no solamente en relación al uso de las TIC, sino también en la formación de la ética que conlleva convertirse en ciudadanos digitales.

Una posible línea futura de investigación sobre las formas de participación en línea de los estudiantes universitarios podría atender a las posibles afectaciones entre los tipos de interacción que realizan con los mecanismos de apropiación para el desarrollo de la competencia de ciudadanía digital vista a través de las construcciones sociales que se desarrollan en el seno de las universidades públicas. Así como el diseño de planes y programas de estudio en las áreas de formación general que contemplen no solo el desarrollo de una alfabetización tecnológica enraizada en el uso óptimo de las herramientas tecnológicas sino expresada en una alfabetización digital que contemple rasgos para el desarrollo del nivel de madurez en el comportamiento ético tecnológico deseable en nuestros estudiantes universitarios en su rol como futuros ciudadanos digitales. 


\section{Agradecimientos}

Investigación subvencionada por CONACYT - 2017-18. (Consejo Nacional de Ciencia y Tecnología de México).

\section{Referencias}

Alanis, José Luis, López Marisela y Olmedo, Gerardo. (2008). Uso e integración curricular de las TICs en la Facultad de Ciencias Biológicas y Agropecuarias de la Universidad Veracruzana. Tercer Foro de Investigación Educativa: Construcción colaborativa del conocimiento, Centro de Formación e Innovación Educativa CFIE, del Instituto Politécnico Nacional. D.F. México.

Arras, Ana María, Torres, Carlos Arturo y Fierro, Luz Ernestina. (2012). Competencias en TIC y rendimiento académico en las universidades autónoma de Chihuahua y Veracruzana. Diferencias por género. México: Pearson.

Arras, Ana María, Torres, Carlos Arturo y García-Valcárcel, Ana. (2011). Competencias en Tecnologías de Información y Comunicación (TIC) en estudiantes universitarios. Revista Latina de Comunicación Social RLCS, (66), 1-26. Recuperado de http://www.redalyc.org/articulo.oa?id=81921340018

Arredondo, Víctor. (1999). Nuevo modelo educativo para la Universidad Veracruzana. Lineamientos para el nivel licenciatura. Propuesta. Recuperado de https://www.uv.mx/afbg/files/2014/05/Nuevo Modelo Educativo Lin.pdf

Ballesta, Javier y Guardiola, Plácido. (2001). El profesorado ante las nuevas tecnologías y los medios de comunicación. Enseñanza: anuario interuniversitario de didáctica (19), 211-258. Recuperado de http://espacio.uned.es/fez/eserv/bibliuned:20456/profesorado nt.pdf

Borromeo, César Augusto y Rámirez, Moisés. (2016). Uso de redes sociales en docentes de lengua inglesa y pedagogía: ¿Diferencias por disciplina? Revista Ensayos Pedagógicos, (Edición Especial), 189-205. Recuperado de http://www.revistas.una.ac.cr/index.php/ensayospedagogicos/article/view/9347

Bringué, Xavier y Sádaba, Charo. (2011). La generación interactiva en Andalucía. Niños y adolescentes ante las pantallas (Colección Fundación Telefónica). Madrid: Foro Generaciones Interactivas.

Campos, Eva y Silván, Aitor. (2012). Democracia digital. El estado de la cuestión. En Irene Ramos y Eva Campos (Coords.), Ciudadanía en 3D: Democracia Digital Deliberativa. Un análisis exploratorio (pp. 53-84). España: Fundación Ideas.

Cano-Correa, Ana María, Quiroz-Velasco, María Teresa y Nájar-Ortega, Rosario. (2017). Jóvenes universitarios en Lima: Política, medios y participación. Comunicar, 53(25), 71 79. Recuperado de https://www.revistacomunicar.com/index.php?contenido=detalles\&numero=53\&articulo $=53-2017-07$ 
Casillas, Miguel Ángel y Ramírez-Martinell, Alberto. (2015). Génesis de las TIC en la Universidad Veracruzana: Ensayo de periodización. México: Productora de Contenidos Culturales Sagahón Repoll.

Crovi, Delia. (2017). Redes Sociales Digitales: Lugar de encuentro, expresión y organización para los jóvenes. México: Ediciones La Biblioteca. Edición de Kindle.

Díez-Gutiérrez, Enrique Javier, Fernández-Rodríguez, Eduardo y Anguita-Martínez, Rocío. (2011). Hacía una teoría política de la socialización cívica virtual de la adolescencia. Revista Interuniversitaria de Formación del Profesorado, 71(25,2), 73-100. Recuperado de http://www.redalyc.org/html/274/27422047005/

Delgado-Salazar, Ricardo, y Arias-Herrera, Juan Carlos. (2008). La acción colectiva de los jóvenes y la construcción de ciudadanía. Revista Argentina de Sociología, 6(11), 272296. Recuperado de http://www.scielo.org.ar/pdf/ras/v6n11/v6n11a14.pdf

Domínguez, Fernando. (2016). Redes sociales digitales y juventud universitaria: Un tema emergente en investigación educativa. Revista Ensayos Pedagógicos, (Edición especial), 55-71. Recuperado de http://www.revistas.una.ac.cr/index.php/ensayospedagogicos/article/view/9340

Domínguez, Fernando y López, Rocío. (2015). Uso de las redes sociales digitales entre los jóvenes universitarios en México. Hacia la construcción de un estado del conocimiento (2004-2014). Revista de Comunicación, 14, 48-69.

Dorantes, Jeysira. (2016). Redes sociales y ciberbullying en la Universidad Veracruzana. Revista Ensayos Pedagógicos, Edición Especial, 169-188. Recuperado de http://www.revistas.una.ac.cr/index.php/ensayospedagogicos/article/view/9346

Ferrés, Joan, Aguaded, Ignacio y García-Matilla, Agustín. (2012). La competencia mediática de la ciudanía española: dificultades y retos. Icono 14, 10(3), 23-42. Doi $\underline{10.7195 / \text { ri14.v10i3.201 }}$

García-Valcárcel, Ana y Arras, Ana. (2011). Competencias en TIC y rendimiento académico en la universidad. Diferencias por género. México: Pearson.

Gavilan, Diana, Martínez-Navarro, Gema y Fernández-Lores, Susana. (2017). Universitarios y redes sociales informativas: Escépticos totales, moderados duales o pro-digitales. Comunicar, 25(53), 61-70. doi: https://doi.org/10.3916/C53-2017-06

Gisbert, Mercé y Esteve, Francesc. (2011). Digital Leaners: la competencia digital de los estudiantes universitarios. La Cuestión Universitaria, (7), 48-59. Recuperado de http://polired.upm.es/index.php/lacuestionuniversitaria/article/view/3359/3423

Gómez, José Alberto. (2013). Redes sociales y comunicación en Internet. Medio ambiente y participación ciudadana en México (Tesis de doctorado). Departamento de Ciencias de la Comunicación. Universidad de Santiago de Compostela, España. Recuperado de http://tesisenred.net/handle/10803/124449 
Granados-Romero, John, López-Fernández, Raúl, Avello, Raidell, Luna-Álvarez, Diego, Luna-Álvarez, Enrique, y Luna-Álvarez, Walter. (2014). Las tecnologías de la información y las comunicaciones, las del aprendizaje y del conocimiento y las tecnologías para el empoderamiento y la participación como instrumentos de apoyo al docente de la universidad del siglo XXI. Medisur, 12(1), 289-294. Recuperado de http://medisur.sld.cu/index.php/medisur/article/view/2751/1452

lbañez, Jesús. (1998). El regreso del sujeto: la investigación social de segundo orden. España: Siglo XXI Editores.

Islas, Octavio y Arribas, Amaia. (2010). Comprender las redes sociales como ambientes mediáticos. En Alejandro Piscitelli, Iván Adaime, e Inés Binder (Coords.), El proyecto Facebook y la postuniversidad. Sistemas operativos sociales y entornos abiertos de aprendizaje (pp. 147-161). Madrid: Ariel / Fundación Telefónica. Recuperado de https://www.fundaciontelefonica.com/arte cultura/publicaciones-listado/pagina-itempublicaciones/itempubli/4/

Livingstone, Sonia, Haddon, Leslie y Görzig, Anke. (2012). Children, risk and safety on the Internet: Research and policy challenges in comparative perspective. Great Britain: The Policy Press.

López, Rocío y Alarcón, Esmeralda. (2016). Jóvenes estudiantes de la Universidad Veracruzana interactuando en red: ¿Diferencias por género? Revista Ensayos Pedagógicos, (Edición Especial), 75-91. Recuperado de http://www.revistas.una.ac.cr/index.php/ensayospedagogicos/article/view/9341

López, Rocío y Anaya, Roberto. (2016). Estudiantes universitarios interactuando en red: ¿nuevos escenarios de interacción, expresión y participación ciudadana? Revista Interamericana de Educación de Adultos, 38(1), 60-90. Recuperado de http://www.crefal.edu.mx/rieda/images/rieda-2016-1/exploraciones2.pdf

López, Rocío. (2016). Jóvenes estudiantes de la Universidad Veracruzana interactuando en red. En Delia Crovi (Coord.), Redes Sociales Digitales: Lugar de encuentro, expresión y organización para los jóvenes (pp. 159-188). México: Ediciones La Biblioteca. Edición de Kindle.

Marini, Verónica, Jácome, Nancy y López, Rocío. (2016). Uso de redes sociales digitales entre estudiantado universitario: Comunicación, socialización y colaboración. Revista Ensayos Pedagógicos, (Edición Especial), 131-149. Recuperado de http://www.revistas.una.ac.cr/index.php/ensayospedagogicos/article/view/9344

Martínez, Karla Paola y Ramírez, Alberto. (2016). Redes sociales en educación superior: Transformaciones tecnológicas de socialización y de colaboración entre estudiantado universitario. Revista Ensayos Pedagógicos, (Edición Especial), 93-111. Recuperado de http://www.revistas.una.ac.cr/index.php/ensayospedagogicos/article/view/9342

Martínez, Raciel D. (2016). Redes sociales y comunicación universitaria: los desafíos de la Universidad Veracruzana en la época de Internet. Revista Ensayos Pedagógicos, (Edición $\quad$ Especial), 37-53. Recuperado de http://www.revistas.una.ac.cr/index.php/ensayospedagogicos/article/view/9339 
Morales, Ana Teresa, Olguín, Pablo Alejandro y Ramírez, Alberto. (2016). Comparativa disciplinar de la comunicación y colaboración del profesorado universitario a través de las TIC. Revista Ensayos Pedagógicos, (Edición Especial), 151-167. Recuperado de http://www.revistas.una.ac.cr/index.php/ensayospedagogicos/article/view/9345

Mossberger, Karen, Tolbert, Caroline J., y McNeal, Ramona S. (2007). Digital citizenship: The Internet, society, and participation. Cambridge, MA: MIT Press.

Ohme, Jakob, Albaek, Erik y de Vreese, Claes H. (2016). Exposure Research Going Mobile: A Smartphone-Based Measurement of Media Exposure to Political Information in a Convergent Media Environment. Communication Methods and Measures, 10(2-3), 135148. Doi $\underline{10.1080 / 19312458.2016 .1150972}$

Ramírez-Martinell, Alberto y Casillas, Miguel Ángel. (2015). Háblame de TIC (Volumen 2: Internet en Educación Superior). Argentina: Brujas-Social TIC.

Ribble, Mike S., Bailey, Gerald D. y Ross, Tweed W. (2004). Digital Citizenship: Addressing Appropriate Technology Behavior. Learning \& Leading with Technology, 32(1), 6-9. Recuperado de https://files.eric.ed.gov/fulltext/EJ695788.pdf

Saorín, Tomás y Gómez-Hernández, José Antonio. (2014). Alfabetizar en tecnologías sociales para la vida diaria y el empoderamiento. Anuario ThinkEPI, 8, 342-348. Recuperado http://eprints.rclis.org/23752/1/Saorin GomezHernandez Thinkepi 2014.pdf

Torres-Gastelú, Carlos Arturo, Angulo-Armenta, Joel, Lagunes-Domínguez, Agustín y SotoOrtiz, José Luis. (2017). Vestiges of a Digital Citizenship in Students of a Mexican University. TOJET. The Turkish Online Journal of Educational Technology, (Special Issue), 920-928. Recuperado de http://www.tojet.net/special/2017 11 1.pdf

Torres-Gastelú, Carlos Arturo y Moreno-Coatzozón, Gustavo. (2013). Inclusión de las TIC en los escenarios de aprendizaje universitario: Diferencias a nivel centro y género. Apertura Revista de Innovación Educativa, 5(1), 48-65. Recuperado de http://www.redalyc.org/pdf/688/68830443005.pdf

Torres-Gastelú, Carlos Arturo y Valencia-Avilés, Luis Alfredo. (2013). Uso de las TIC e Internet dentro y fuera del aula en alumnos del sexto grado de primarias públicas en Veracruz. Apertura Revista de Innovación Educativa, 5(1), 108-119. Recuperado de http://www.udgvirtual.udg.mx/apertura/index.php/apertura/article/view/381

Torres-Gastelú, Carlos Arturo y Arras-Vota, Ana María. (2011). Percepción de estudiantes de educación superior sobre sus competencias en las TIC entre los colectivos de estudiantes de licenciaturas afín de sistemas en las universidades Autónoma de Chihuahua y Veracruzana. Apertura Revista de Innovación Educativa, 3(2), 90-107. Recuperado

de http://www.udgvirtual.udg.mx/apertura/index.php/apertura/article/view/207/222

Universidad Veracruzana, Secretaría de la Rectoría, Dirección de Planeamiento Institucional. (2016). Numeralia. Recuperado de https://www.uv.mx/numeralia/files/2016/05/Numeralia.pdf 
Universidad Veracruzana. (2009). Cuarto informe de labores 2008-2009. Recuperado de https://www.uv.mx/universidad/doctosofi/informe2008-2009/Cuartolnforme08-

09(sintesis).pdf

Valerio-Ureña, Gabriel y Valenzuela-González, Ricardo. (2011). Redes sociales y estudiantes universitarios: del nativo digital al informívoro saludable. El profesional de la información, 20(6), 667-670. Recuperado de http://catedra.ruv.itesm.mx/bitstream/987654321/759/1/Redes\%20sociales\%20y\%20est udiantes\%20universitarios.\%20Del\%20nativo\%20digital\%20al\%20informivero\%20salud able.pdf

Viché, Mario. (2015). El empoderamiento de los ciudadanos Internet. Ponencia presentada en el Congreso sobre Movimientos Sociales y TIC, Sevilla, España. Recuperado de https://idus.us.es/xmlui/handle/11441/44117

Yuste, Bárbara. (2015). Las nuevas formas de consumir información de los jóvenes. Revista de Estudios de Juventud, (108), 179-191. Recuperado de https://dialnet.unirioja.es/servlet/articulo?codigo $=5195624$ 